Ebisu Ebisu

Études japonaises Études japonaises

47 | printemps-été 2012

Catastrophes du 11 mars 2011, désastre de

Fukushima : fractures et émergences

\title{
Convertir les « allergiques à l'atome » : la promotion du nucléaire au Japon
}

Relieving Japan's "Nuclear Allergy": the Promotion of Atomic Power in Japan 「核アレルギー」の日本人を回心させる日本の原子力推進

\section{Mathieu Gaulène}

\section{OpenEdition}

\section{Journals}

Édition électronique

URL : http://journals.openedition.org/ebisu/334

DOI : 10.4000/ebisu.334

ISSN : 2189-1893

Éditeur :

Institut français de recherche sur le Japon (UMIFRE 19 MAEE-CNRS), Maison franco-japonaise

\section{Édition imprimée}

Date de publication : 1 juin 2012

Pagination : 107-118

ISSN : 1340-3656

Référence électronique

Mathieu Gaulène, "Convertir les « allergiques à l'atome » : la promotion du nucléaire au Japon », Ebisu [En ligne], 47 | printemps-été 2012, mis en ligne le 04 avril 2014, consulté le 02 mai 2019. URL : http:// journals.openedition.org/ebisu/334; DOI : 10.4000/ebisu.334 


\title{
Convertir les « allergiques à l'atome » La promotion du nucléaire au Japon
}

\author{
Mathieu GAULÈNE
}

Quelques jours après le début de l'accident nucléaire de Fukushima, les Japonais virent apparaittre sur les écrans de télévision une publicité particulièrement sobre de la Compagnie d'électricité de Tokyo, intitulée "Les excuses de Tepco ». On n’y vantait ni la sécurité de la centrale de Fukushima, ni le caractère propre de l'énergie nucléaire comme cela avait pu être le cas avant. Sur fond blanc, Tepco s'excusait des " fuites radioactives " provoquées par l'accident de Fukushima auprès « des riverains de la centrale, de la population du département de Fukushima et de l'ensemble de la société japonaise ". " Nous nous efforçons de tout remettre en ordre ", ajoutait la compagnie d'électricitél. Depuis, Tepco s'est abstenu de produire des publicités faisant la promotion de l'énergie nucléaire.

Par la suite, Tepco décida de fermer définitivement les portes de son Musée de l'électricité (Denryokukan 電力館), immense complexe implanté en plein cœur de Shibuya² (photo 1). Sur sept étages, ce musée proposait gratuitement aux visiteurs des expositions et des événements divers autour du thème de l'énergie nucléaire entre autres. Au sujet de cette fermeture,

\footnotetext{
\ Mathieu Gaulène est journaliste, spécialiste du Japon. Il réside à Tokyo. Diplômé de l'IEP d'Aix-en-Provence et de Sciences Po Paris, en master Asie, il a notamment rédigé un mémoire sur le mouvement antinucléaire japonais.

1. Voir : <http://www.youtube.com/watch?v=NiJylt2_9JY\&feature=related> (N.D.L.R. : dernière consultation le 10 avril 2012).

2. Le site internet de ce musée a également disparu peu après sa fermeture : <http:// www.denryokukan.com/index.html>.
} 


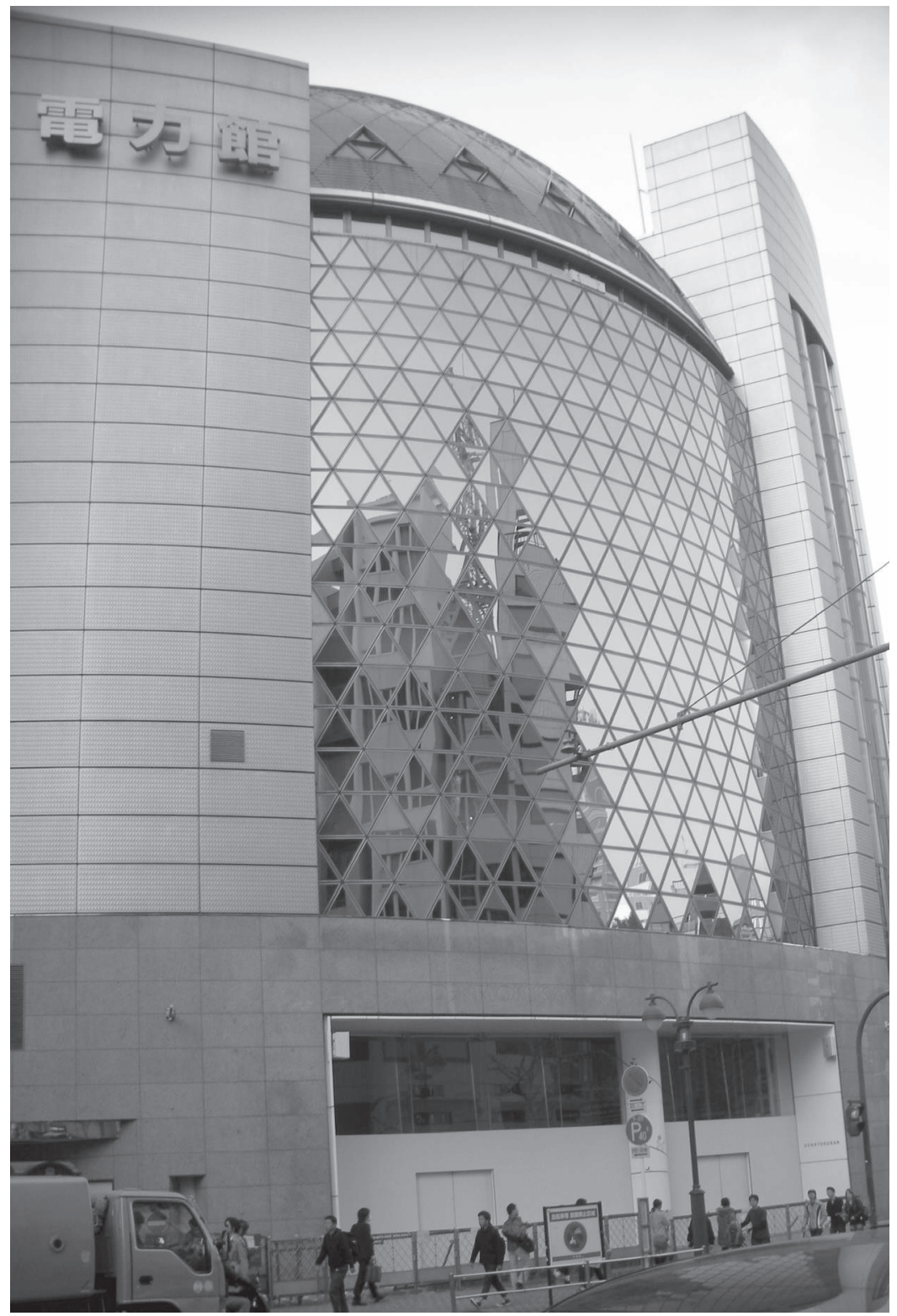

Photo 1 Le musée de l'électricité de Tepco, Shibuya, novembre 2011. 


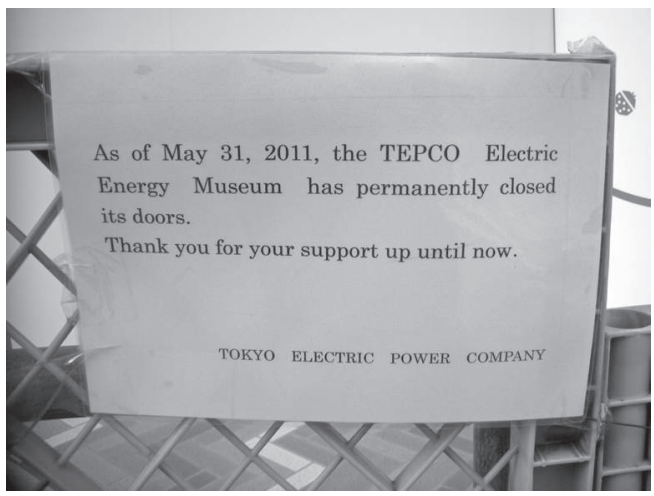

Photo 2 Message d'information à l'entrée du musée de l'électricité de Tepco, novembre 2011.

Tepco n'a pas beaucoup communiqué. À l'extérieur du bâtiment, sur les grilles fraîchement installées, de simples écriteaux expliquent en japonais et en anglais que "le musée a fermé définitivement ses portes le 31 mai 2011 " (photo 2). Un autre précise que le site reste gardé par des caméras de vidéo-surveillance, et les jours de manifestations, l'entrée est protégée par des cars de police.

Ces deux exemples ne sont pas anodins. Publicités et musées du nucléaire (genshiryoku pīàru sentā 原子力PRセンター) sont au Japon les moyens les plus utilisés par le lobby de l'industrie nucléaire pour faire la promotion de cette énergie. Ce lobby peut être défini par l'alliance entre le METI, et plus particulièrement l'Agence des ressources naturelles et de l'énergie (Shigen enerugī chō 資源エネルギー庁), les différentes entreprises liées à la construction de centrales ou à leur entretien et les compagnies d'électricité privées qui gèrent le parc nucléaire japonais. Promouvoir le nucléaire après Fukushima est toutefois devenu complexe et pour l'heure le lobby nucléaire préfère faire profil bas. De plus, les journaux ou les chaînes de télévision auraient bien du mal à accepter aujourd'hui des publicités vantant la sûreté des installations nucléaires.

Il n'en a pourtant pas toujours été ainsi et pendant plus d'un demisiècle les Japonais ont subi un déluge de propagande sur le nucléaire. Les taxes diverses, les subventions gouvernementales et la création d'emplois dans des régions rurales ont pu être un argument convaincant pour faciliter 
l'installation de centrales. Mais le lobby nucléaire japonais s'est toujours évertué à s'attaquer à la racine du problème en changeant les cœurs et les esprits (Aldrich 2008).

\section{Le 26 octobre, « journée du nucléaire » au Japon}

Pour contrer l'image négative du nucléaire chez les Japonais, le gouvernement met en place dès 1964, la " journée de l'énergie nucléaire " (genshiryoku no hi 原子力の日). Elle commémore le lancement le 26 octobre 1963 du premier réacteur expérimental à Tōkai-mura, dans le département d'Ibaraki. Chaque année sont organisés, par exemple, des concours d'essais mettant en valeur les mérites et la sécurité de l'énergie nucléaire ou des concerts gratuits. La presse et la télévision se font le relais de publicités décrivant les bienfaits du nucléaire ${ }^{3}$.

Pour faire l'apologie de l'énergie nucléaire, tous les moyens sont bons : pour la journée du nucléaire du 26 octobre 1978, l'Agence japonaise pour la science et la technologie (Kagaku gijutsu chō 科学技術庁) diffuse un poster montrant une jeune japonaise nue, se cachant les seins avec les bras (Horie 1984 : 81) (photos 3 et 4). Une formule énigmatique « allergique à l'énergie " (enerugī arerugī エネルギー・アレルギー) fait référence à l'expression " allergique à l'atome » (kaku arerugi 核アレルギー). Dès le début des années 1960, les promoteurs du nucléaire ont parlé de "l'allergie des Japonais à l'atome " (Nihonjin no kaku arerugī 日本人の核アレルギー), pour dénigrer la peur supposée irrationnelle du nucléaire. Cette affiche joue ici sur le mot " énergie " en associant l'énergie sexuelle qui se dégage de l'image, à l'énergie atomique. Une façon sans doute de culpabiliser le passant et d'attiser chez lui une frustration.

Le choix des mots révèle de quelle manière les "nucléocrates ${ }^{4}$ " (Simonnot 1978) entendent communiquer au sujet du nucléaire. Le terme

3. Voir l'article de Tino Bruno, «La promotion du nucléaire civil dans la presse quotidienne japonaise " dans le présent numéro (N.D.L.R), ainsi que Daniel P. Aldrich (2008: 125).

4. Cette expression permet de rassembler dans un même groupe l'ensemble des partisans du nucléaire issus de l'industrie, de l'administration et du monde politique ou universitaire. 

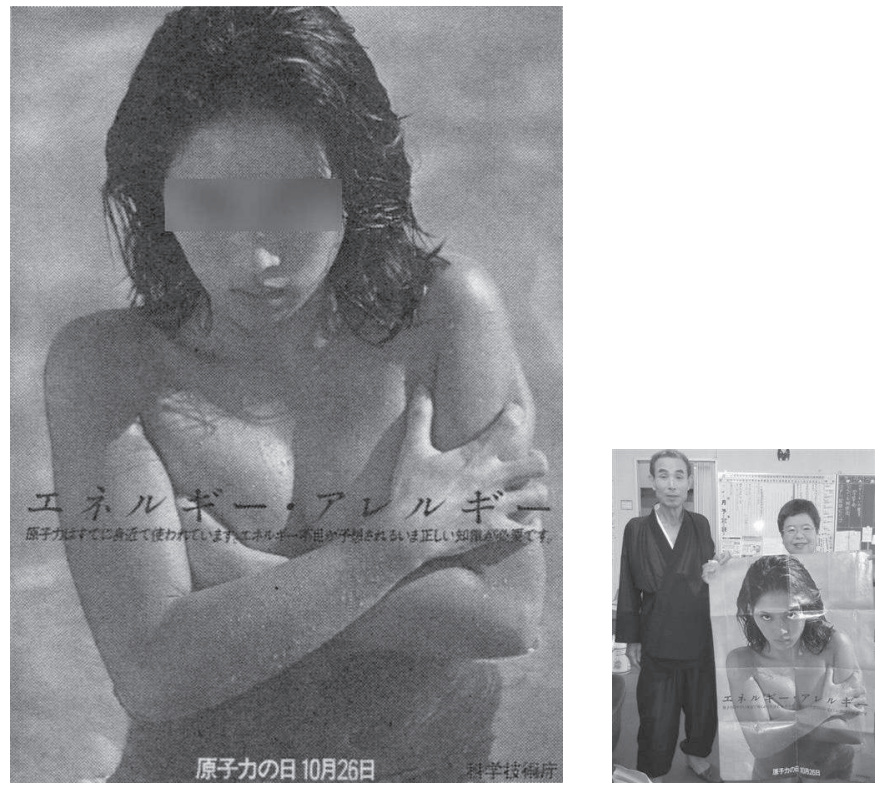

Photo 3 (ci-dessus à gauche) Poster « allergique à l'énergie » conçue par l'Agence japonaise pour la Science et la Technologie à l'occasion de la « journée de l'énergie nucléaire », 1978.

Photo 4 (ci-dessus à droite) L'affiche de la journée du nucléaire, tenue par Mme Miyagawa Emiko 宮川えみこ, députée communiste du département de Fukushima et M. Hayakawa, militant antinucléaire de Fukushima, juillet 2011.

de « recyclage » (risaikuru リサイクル en japonais) utilisé parfois à la place du mot « cycle » (saikuru サイクル), est connoté très positivement, car c'est le même mot qui est utilisé pour le recyclage du plastique ou du verre. Or le " recyclage " des déchets nucléaires, dans ses objectifs comme dans son processus, n'a pas grand-chose en commun avec le recyclage des déchets ménagers. Katō Hideki 加藤秀樹, qui participa à des réunions d'explication (setsumei kai 説明会) sur l'usine de retraitement de Rokkasho-mura ${ }^{5}$,

5. Une partie de cet article s'appuie sur un mémoire de recherche consacré au mouvement antinucléaire japonais et à la lutte contre l'usine de retraitement de Rokkasho-mura 


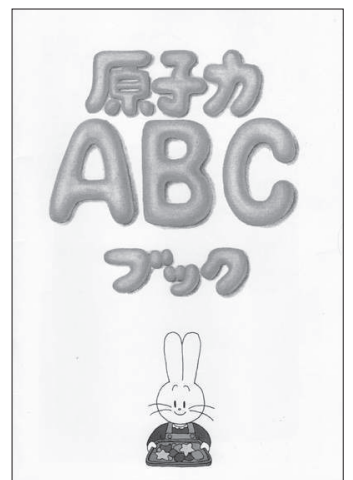

Fig. 1

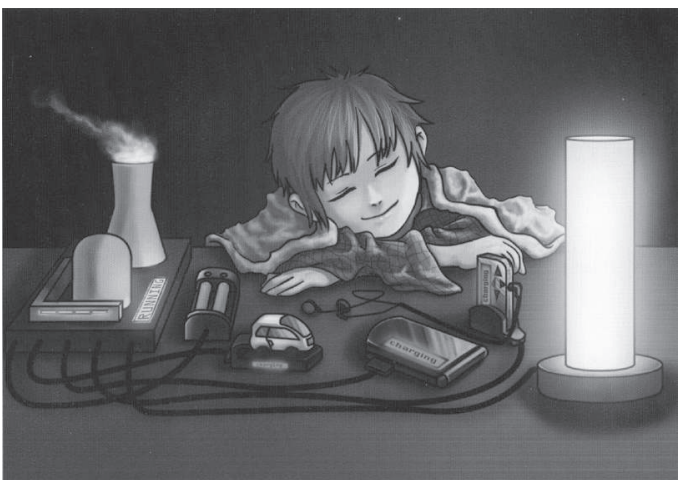

Fig. 2

explique ainsi que la simple utilisation du mot recyclage avait retourné et convaincu l'assemblée des personnes présentes ce jour-là, qui étaient pourtant initialement hostiles à la construction de cette usine ${ }^{6}$.

\section{Spots télévisés et faux documentaires}

Très rapidement, c'est cependant par la télévision que les compagnies d'électricité se sont lancées dans la promotion du nucléaire. Il y est invariablement présenté comme une énergie sûre et propre, sans rejet de $\mathrm{CO}_{2}$.

Mais les entreprises ne se contentent pas d'apparaître aux interstices des programmes télévisés. Elles vont aussi jusqu’à produire des programmes entiers. Ainsi en 2009 était diffusé sur la chaîne BS Japan, un documentaire consacré au village de Rokkasho-mura. Un petit village ayant la

(Gaulène 2009).

6. Katō Hideki est professeur à l'université Keiō et spécialiste des NPO (Non-Profit Organisation). Il est président également de deux think tanks, Japan Initiative (Kōsō Nippon 構想日本) et The Tokyo Foundation (Tōkyō zaidan 東京財団), branche de la très controversée The Nippon Foundation (Nippon zaidan 日本財団), ex-Fondation Sasakawa. Sur ce sujet, voir Gaulène (2011). Partisan critique de l'énergie nucléaire, Katō Hideki est opposé à la construction de l'usine de retraitement de Rokkasho-mura (entretien, 17 mars 2009). 


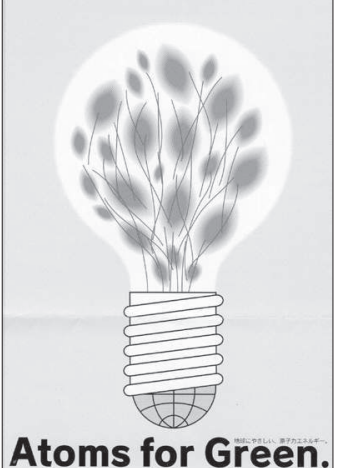

Figures 1, 2 (ci-contre) et 3

La Japan Atomic Energy Relations Organization (JAERO) ou Fondation pour la promotion de la culture du nucléaire au Japon (Nihon genshiryoku bunka shinkō zaidan 日本原子 力文化振興財団) promeut l'énergie nucléaire par le biais de campagnes d'affichages publicitaires, de conférences et de brochures.

Certaines sont spécialement conçues pour les enfants, comme cet «ABC du nucléaire » (Genshiryoku è bī shïbukku) (fig. 1).

Elle organise chaque année un concours d'affiches visant un public large pour la promotion du nucléaire (fig. 2 et 3 : "Atoms for Green », premier prix 2009) et publie chaque mois un mensuel au titre évocateur, Genshiryoku bunka, la «culture du nucléaire ».

Fig. 3

particularité d'accueillir le plus grand complexe nucléaire du Japon et une toute nouvelle usine de retraitement des combustibles usés. Le générique révèle que le programme était sponsorisé par la Fédération des compagnies d'électricité (FEPC, Denki jigyō rengō kai 電気事業連合会) dont le rôle est de financer des programmes télévisés et radiophoniques faisant la promotion du nucléaire, souvent de manière détournée puisque le sponsor est annoncé en fin de reportage.

Ce documentaire décrivait la vie paisible des habitants des différents hameaux de la commune de Rokkasho-mura. Les gens élèvent des vaches, vont à la pêche, un autre a ouvert un restaurant italien il y a deux ans - ce qui donne lieu à un gros plan sur un plat que goûte la journaliste, passage obligé de toute émission de télévision japonaise. L'usine de retraitement est évoquée dès le début du reportage, sans équivoque, mais rapidement le message diffusé par le reportage est limpide : "Tout va bien à Rokkashomura, où il fait bon vivre ».

Le documentaire est entrecoupé de spots de publicité de la FEPC vantant les mérites du nucléaire et dont voici un exemple. Un court dessin animé met en scène un kappa géant sortant brusquement d'un lac-comment ne pas penser à Godzilla ici ? La petite famille en ballade est effrayée : «Ah, ça sort !" (deta! 出た!) mais le monstre-nucléaire les rassure : "Non, il n’y a rien qui sort » (detemasen 出てません), " les éoliennes, les centrales solaires mais aussi les centrales nucléaires ne rejettent pas de $\mathrm{CO}_{2}$ lorsqu'elles sont en fonctionnement ». 


\section{Contraindre avec pédagogie : les réunions d'explication}

Enfin, le lobby nucléaire a recours à un autre instrument qui s'adresse plus particulièrement aux riverains d'une centrale : les réunions d'explications (setsumei kai). Il s'agit en somme, à l'instar de la procédure du débat public en France, de convaincre les citoyens d'une décision sur laquelle ils n'ont aucune prise, tout en leur donnant l'illusion d'en être les co-artisans. Cet instrument est cependant d'une efficacité limitée car ces réunions servent de tribune aux opposants au nucléaire. De fait, elles se transforment souvent en un dialogue de sourds.

Les promoteurs du nucléaire vont parfois plus loin en tentant de s'immiscer dans le maillage du réseau local. À Rokkasho-mura, la Japan Nuclear Fuel Limited (JNFL, Nihon gennen 日本原燃) procède de deux manières comme nous l'expliquait Serizawa Shun.ichi 芹沢俊一, chef du département des relations publiques :

« Nous avons mené deux activités. La première était d'aller faire deux fois par an du porte-à-porte chez tous les habitants du village de Rokkasho, pour leur expliquer en quoi consiste notre entreprise, expliquer ce que sont les usines du cycle de combustible. La deuxième activité qui a été menée plus particulièrement par le département des relations publiques de la JNFL consistait à rendre visite aux “leaders”, c'est-à-dire aux politiciens locaux et aux membres de l'assemblée municipale, pour leur faire comprendre et leur indiquer la bonne direction ${ }^{7}$."

Les "leaders " ont effectivement été largement utilisés car il s'agissait pour les compagnies d'électricité et le gouvernement de convaincre prioritairement ceux qui ont le pouvoir de décision. Rien ainsi n'a été laissé au hasard et l'État japonais a reçu l'aide d'Areva (ex-Cogema), chargée du transfert de technologie pour la construction de l'usine de retraitement. La direction de l'usine de La Hague envoya par exemple un maire français pro-nucléaire au village de Rokkasho, " pour persuader les habitants qu'il est aisé de vivre à proximité d'un tel établissement " (Zonabend 1989 : 16). Puis, on a fait faire le voyage en sens inverse à des habitants de Rokkashomura, comme nous le rapporta le conseiller nucléaire de l'ambassade de France à Tokyo :

7. Entretien avec Serizawa Shun.ichi et Sasaki Yoshiaki 佐々木義明, 26 mars 2009. 
"On envoie en fait ce qu'on appelle des "relais d'opinions", c'est-à-dire par exemple les femmes des pêcheurs ou des cultivateurs de betteraves de Trifouillis-les-Oies version Japon, on leur fait visiter les sites en France pour leur montrer que tout va bien $^{8}$."

Pourquoi envoie-t-on des femmes ? Parce que ce sont les femmes, et surtout les mères, qui sont à la tête du mouvement antinucléaire nippon. Un sondage de la Japan Atomic Industrial Forum (JAIF, Nihon genshiryoku sangyō kyōkai 日本原子力産業協会) de 2008 montre d'ailleurs qu’à la question "l'énergie nucléaire est-elle nécessaire ? ", 27,4\% des hommes répondent " oui " contre $9 \%$ pour les femmes (JAIF 2008 : 24). Depuis le 11 mars 2011, on retrouve beaucoup de femmes à la tête des mouvements antinucléaires, et notamment des mères du département de Fukushima venues camper sous les fenêtres du METI ${ }^{9}$.

\section{Un musée « spécialement conçu pour les mères et les enfants »}

L'une des dernières pierres venant consolider l'édifice de la promotion du nucléaire au Japon réside dans les nombreux "musées du nucléaire " qui parcellent l'archipel. Chaque compagnie d'électricité en possède au moins un, servant surtout à convaincre les nouvelles générations vivant près d'une centrale du bien-fondé de cette industrie. À Rokkasho-mura, la JNFL ne déroge pas à la règle avec son "Musée des combustibles nucléaires " (Rokkasho gennen pīāru sentā 六ヶ所原燃PRセンター) construit par l'architecte Kurokawa Kishō 黒川紀章. Premier détail frappant : le musée est gratuit. Sur des écrans, les mascottes du musée expliquent gaiement le « cycle du combustible nucléaire ", i.e. son retraitement. Puis vient l'attraction principale : du deuxième au premier étage on peut suivre en effet toutes les étapes du retraitement. En modèle réduit, une barre de combustible usée est retirée d'une petite piscine, puis insérée dans une zone invisible où

8. Entretien téléphonique avec Pierre-Yves Cordier, directeur du SNAFT (Service nucléaire de l'ambassade de France à Tokyo), 11 décembre 2008.

9. Voir la contribution de Christine Lévy dans le présent volume [N.D.L.R]. 
les barres sont cisaillées. On descend d'un étage et les morceaux arrivent avec grand bruit dans un tuyau transparent pour tomber dans une cuve d'acide nitrique représentée par un autocollant jaune fluo. Enfin vient le moment où l'uranium et le plutonium sont séparés, dans un festival de loupiotes rouges et vertes ressemblant plus à l'univers de Star Trek qu'à une usine de retraitement. Mais sans doute faut-il insuffler du rêve pour obtenir l'adhésion.

Les motifs de ce manège enchanté nous sont fournis quelques minutes plus tard alors que nous remarquons dans un coin des petites tables pour enfants avec des jouets et des peluches. Très étonné, nous en demandons la raison à Sasaki Yoshiaki du département des relations publiques de la JNFL. "Ce musée est spécialement conçu pour les mères et leurs enfants » explique-t-il. Les femmes et leurs enfants, la future génération de Rokkasho-mura, font l'objet d'une attention toute particulière, à la mesure de la menace qu'ils représentent pour l'industrie nucléaire.

\section{Le lobby nucléaire japonais a-t-il dit son dernier mot ?}

La question mérite d'être posée. Certes, le cas de Tepco pourrait donner l'impression que le temps des publicités dans la presse ou à la télévision est révolu. Mais la promotion du nucléaire a-t-elle complètement cessé au Japon ? La compagnie d'électricité du Chūbu continue depuis le 11 mars 2011 à diffuser des publicités où certes le nucléaire n'est pas mentionné, mais dont les slogans soulignent l'importance de l'électricité dans le quotidien des Japonais. La FEPC continue discrètement de son côté à financer certains programmes de radio. Surtout, le poids du silence ou du déni de la réalité de la contamination et des conséquences sanitaires sur la population et les « liquidateurs » est toujours plus pesant. La plupart des grands médias ont bénéficié pendant des années de solides recettes engrangées pour faire la publicité des compagnies d'électricité ${ }^{10}$.

10. À l'exception notable des quotidiens Mainichi shinbun, Tökyō shinbun et Akahata shinbun. 
Revenons sur le musée de Rokkasho-mura pour donner une illustration frappante de ce déni. Le 17 mars 2011, celui-ci avait fermé ses portes « en raison des difficultés d'approvisionnement en électricité suite à la catastrophe de l'est du Japon ». Pas une ligne n'évoquait l'accident nucléaire de Fukushima. Ce musée aurait pu connaître le même destin que le Denryokukan de Shibuya, mais il a finalement rouvert ses portes, le 11 avril $2011^{11}$. Depuis, les visites des écoliers de Rokkasho-mura ont repris, comme si rien ne s'était passé.

La question d'une sortie du nucléaire fait partie aujourd'hui du débat public au Japon. Sur la défensive, le "village nucléaire " (genshiryoku mura 原子力村) a fait le choix du silence pour le moment, qui est en soi une forme de non-communication sur les événements. Nul doute cependant qu'une fois la vague de protestation passée, la propagande du nucléaire, arme non-négligeable, reprendra son cours. Car la sortie ou non du nucléaire sera déterminée par l'issue du rapport de force qui s'est instauré depuis le 11 mars entre les promoteurs du nucléaire et une population qui y est majoritairement opposée.

Décembre 2011

11. Site internet : <http://www.6prc.co.jp/index.html> (N.D.L.R. : dernière consultation le 10 avril 2012). 


\section{Bibliographie}

\section{ALDRICH Daniel P., 2008}

Site Fights. Divisive Facilities and Civil

Society in Japan and the West, Ithaca,

Cornell University Press.

\section{GAULÈNE Mathieu, 2009}

Mouvement antinucléaire et changement démocratique au Japon. Le cas de la mobilisation contre l'usine de retraitement de Rokkasho-mura, Paris, IEP.

\section{GAULÈNE Mathieu, 2011}

«De quoi la Fondation Sasakawa est-elle le nom ?», Nonfiction.fr.

\section{HORIE Kunio 堀江邦夫, 1984}

Genpatsu jipushī 原発ジプシー (Les Gitans du nucléaire), Tokyo, Gendai shokan 現代書館.

\begin{abstract}
JAIF, 2008
Enerugī ni kan suru ishiki chōsa エネルギー に関する意識調査 (Enquête d'opinion sur l'énergie).
\end{abstract}

SIMONNOT Philippe, 1978

Les Nucléocrates, Grenoble, Presses universitaires de Grenoble.

ZONABEND Françoise, 1989

La presqu'île au nucléaire, Paris, Odile Jacob. 